\title{
IDEAL KIOSK PLACEMENT IN UP DILIMAN FOR EQUAL PROBABILITY OF CUSTOMER TURNOUT
}

\author{
K. A. Cabello ${ }^{1}$, M. A. A. Manabat ${ }^{1}$, M. B. A. Zamora ${ }^{1}$, A. C. Blanco ${ }^{1,2}$ \\ ${ }^{1}$ Department of Geodetic Engineering, University of the Philippines Diliman \\ 2 Training Center for Applied Geodesy and Photogrammetry, University of the Philippines Diliman \\ keithann.cabello@gmail.com,mrieann24@gmail.com,mizamora@up.edu.ph, acblanco@up.edu.ph
}

\section{Commission IV}

KEY WORDS: business, permutation, combination, OpenStreetMap, Network Analysis, closest facility, DEM, optimal location, GIS

\begin{abstract}
:
Kiosks scattered around the UP Diliman campus have always been playing a significant role in shaping the present culture in the university. However, competition is inevitable among owners. Some have even lowered their prices to attract more customers and increase sales and profits. One of the reasons why some kiosks earn more than others is because of the location of the kiosks and their distribution within the campus. In this study, accessibility, walkability and site availability are the main factors that were considered to create an ideal kiosk distribution model. First, road points within an area with $10 \%$ slope grade and below were only considered to obtain optimum gradient that requires the least energy. Road points that are within a buffer of 5 meters from waiting sheds and parking lots and 10 meters from buildings were accepted to consider the accessibility of each point. Closest Facility Tool (CFT) under Network Analysis was used to identify road points within 200 meters of each building. These points were considered as good candidate locations for kiosks. CFT was used again to identify buildings within 200 meters from each candidate point. Potential customer count (PCC) per building was reduced when a canteen is within 200 meters. Lastly, the PCC multiplied by the inverse of the distance was used as weight to identify the area that will result in equal demands of customers. Python code was used to iteratively identify the combination of road points that have relatively equal weights. A map showing all 20 current kiosks in their ideal locations and a list of landmarks surrounding each kiosk were produced. Results show that, ideally, five kiosks should be located near Palma Hall, which has the largest population count. Two kiosks should also be placed between the Law Center and Asian Center, between the Institute of Biology and the National Institute of Molecular Biology and Biotechnology, and near College of Arts and Letters, Institute of Electrical and Electronics Engineering (IEEE) and College of Human Kinetics (CHK). Finally, only one kiosk each should be placed near buildings such as Melchor Hall, the National Institute of Physics (NIP), the National Institute of Geological Sciences (NIGS), the Institute of Civil Engineering (ICE) and Albert Hall.
\end{abstract}

\section{INTRODUCTION}

\subsection{Background of the Study}

Since time immemorial, kiosks located all around the campus of UP Diliman has always been a trademark and had its fair share on the university culture. However, owners of small businesses like these kiosks are greatly affected by competition, meaning some of them leaned into cutting price ranges in order for them to gain more customers, thus, making more profit. But in reality, it is observed that lowering the selling price of their products is not the main reason why each kiosk earns more than the others. Location of the kiosk itself and how well dispersed are they from one another plays key to getting equal customers.

According to Garner (2013), there are seven (7) top factors that impact the success of one's business's location. These are: (1) accessibility - how easy it is to be seen and to go to, (2) competition - presence of nearby competitor requires extra effort to retain interest, (3) business environment - other present businesses around and their potential impacts to one's business, (4) resources - access to urgent services (i.e. postal services, telephone, internet, banking and security services), (5) site availability and regulations - all municipal regulations like licences and taxes involved in the business location, (6) costs rent of the place and (7) physical layout - interior and exterior of the location.

Issues concerning the current locations of the kiosk have been verified by Ms. Ellen, President of the Samahang Manininda sa UP Campus which includes a possible relocation of all kiosk from their current spaces, which would greatly cause their incomes to decrease.

\subsection{Problem Statement}

This project aims to answer the question "Where are the ideal locations of kiosk in UP Diliman such that customer turnout for each one equates?".

\subsection{Objectives}

The objectives of this study are the following:

1. To identify potential number of passersby along roads; 
2. To identify accessible points considering the mode of travel as walking; and

3. To identify the locations where customer turnout for each is equal.

\subsection{Scope and Limitations}

The study will only consider factors relating to accessibility and site availability based from the article written by Garner (2013). To measure accessibility to get the most optimal locations for kiosks, Dubuc (2007) listed three (3) factors which are distance, time, and cost. The optimal model is based on minimum distance and/or time of travel to find the least cost path between points on a network which are the two main variables used for this study.

Another factor to be considered is walkability, which measures how friendly an area is to walking. This includes pathways that are more accessible to buildings. (TDM Encyclopedia, 2017). In this setup, paths to be chosen are ones that make kiosks accessible to people. Since the university has its own transportation system, waiting sheds are scattered making areas near them as the most optimal candidates in the location of kiosks.

Lastly, a $10 \%$ slope in the area is also considered to obtain an optimum gradient that only requires the least energetic cost (Casinos \& Saborit, 2015).

\section{REVIEW OF RELATED LITERATURE}

\subsection{Network Analysis}

\subsubsection{Service Area}

Service area is one of ArcGIS Network Analyst extension that creates a region that encompasses all accessible streets within a specified impedance. This helps users evaluate how much land, how many people, or how much of anything else is within the neighborhood or region. (ESRI,n.d.)

In creating the service area analysis layer, there are six (6) classes involved, namely: (1) Facilities, (2) Lines, (3) Polygons, (4) Point Barriers, (5) Line Barriers, and (6) Polygon Barriers. Facilities class stores the network locations currently analyzed. Lines class stores resultant service areas as line features and represents network edges that can be reached within the set impedance. (ESRI,n.d.) Polygon class, on one hand, works the same as the lines class but instead of storing created service area to linear features, it stores it to polygons.

Three barrier classes conclude to one parameter which are the Restrictions involved. By the user's set impedance or tolerance, restriction attributes prohibit roads (lines) and buildings (polygons) from being classified in the service area.

\subsubsection{Closest Facility}

Closest facility solver displays the optimal routes between set facilities to incidents. This identifies the cost and direction of travel between points and determines which of these are nearest to one another. (ArcGIS Pro, n.d.)

In creating the closest facility layer, there are three (3) feature classes involved, namely: (1) Facilities, (2) Incidents and (3) Routes. Facilities feature class stored the network locations either used as the starting or ending points. Incidents, on one hand, works the same way as the facilities feature class but must always be on the opposite end of a route from the connected facility. Both classes can start or end the route class, which is the resulting route/s of the analysis.

Same as the service area, users can input barriers to prohibit certain regions or restrict them if necessary.

\subsubsection{Buffer}

Buffer is a zone or region that bounds area by a specified maximum distance and encompasses all nodes and elements present along with the segments of a starting object. In the Buffer tool of ArcGIS, a buffer distance and type of method are usually the parameters considered. Generally, buffer distance can be a fixed value --meaning distance is constant throughout set value or a field containing numeric values -- meaning distances are dependent on the set field values which results in various buffer widths.

In terms of method, the Buffer Tool can let users determine how the buffer is going to be constructed. There are two basic methods, namely: (1) Euclidean and (2) Geodesic buffering. Euclidean buffers measure the distance between two points in a two-dimensional Cartesian plane. This type of buffering is more common and works well in analyzing distances of features in a certain projected coordinate system where areas and shapes of features can be distorted.

Geodesic buffers accounts for the true shape of the earth. Compared to straight lines for Euclidean, geodesic distances are calculated by measuring two points on a curved surface (geoid) preserving the shape regardless of the input coordinate system. (ESRI, n.d.)

\subsection{Finding Optimal Locations using GIS}

GIS-based methods have always been used and applied in different studies all related to finding optimal locations used for efficiency purposes. Tapping on the business side, location is very important as it links many companies together and paves possible solutions and a certain understanding of the state of the business itself by analyzing data such as sales, profit-driving services, customers' characteristics, and more.

For instance, Gordon (2017) conducted a study about successful franchise businesses such as Starbucks and Dunkin Donuts which have been analyzed to know how these companies place their business in beneficial locations and the techniques they have used to do so. 
Upon researching Starbucks and Dunkin Donuts GIS strategies, it is found that customer demographics play a major role and that with the support of data from the use of ArcGIS or Tango Analytics, these businesses ensured the best location was chosen by factors like traffic flow (both foot and automotive), local crime rates, local competitors, and brand awareness.

Finding market zones in a study by Bojovic, et. al (2005) for retail business has also used GIS tools to strongly support that to maintain a positive feedback on business despite being under high costs and the maintenance of a quality service, choosing retail outlet location based on the market potential of the store highly affects the business.

Not only is GIS used in businesses but also in hazard identification and risk management. Kar and Hodgson (2008) modeled a site suitability map of emergency evacuation shelters in Florida due to an increase in the number of hurricanes and other coastal hazards in the region. This reduces mortality and provides emergency services by evacuating people from the hazard zone.

Feng, Jin Feng and Wen sheng (2007) were also able to conduct an analysis of seismicity of China using all earthquake records available dating back to B.C. 3000 . The group was able to obtain information layers such as maps of earthquake intensity with expected damage and other possible hazards involved once any earthquake hits China. With the use of GIS, a spatial-temporal pattern of the seismic hazard was mapped providing identification to regions repeatedly affected by seismic events and a further extension of assessment to other more natural risks. Furthermore, study helps identify the least areas to build infrastructures and which areas need more attention once help is needed.

\section{MATERIALS AND METHODS}

\subsection{Materials}

Below are lists of materials and datasets collected from OpenStreetMap and populations count from UP CRS, UP HRDO (Human Resources Development Office) and UP OSH (Office of Student Housing).

\subsubsection{Shapefiles}

Footprint of UP Diliman Campus

Roads present in the said campus

DEM SRTM (5m resolution)

\subsubsection{Count data}

Number of students per building

Number of faculty and staff per building

Number of dormers

Number of sheds

Number of canteens

\subsubsection{Software}

ArcGIS 10.5

\subsubsection{Hardware}

Dell Inspiron 151588

HP Pavilion x 360

\subsection{Methodology}

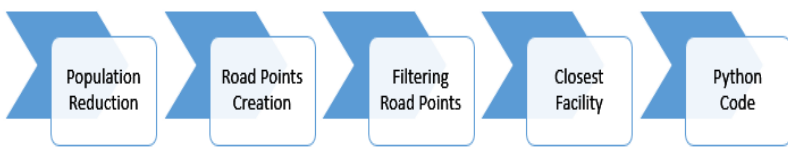

Figure 1. Flow chart of the process in finding points in UP Diliman that would give the least difference in customer probability.

\subsubsection{Reducing population count by considering canteen presence in the area.}

The weights of each canteen are computed using the population count of its neighboring buildings.

From here, each weight is then distributed to the buildings around it. Buildings with two or more nearby canteens will show the higher value of weight than the rest of those buildings distant from them.

After the accumulation of total canteen weights per building, population is then reduced.

\subsubsection{Road points Creation}

Road polylines were converted to raster with $10 \mathrm{~m}$ resolution and were then converted to points, thus having hundreds of road points that are $10 \mathrm{~m}$ apart

\subsubsection{Filtering road points}

\subsubsection{Slope}

To filter out these road points, one parameter used was the slope. According to DEM file was converted to slope values using the slope tool in ArcGIS. The slope raster was then reclassified to get areas with a slope of $10 \%$ and below only. This reclassified slope raster was then converted to vector. Road points that are not intersected by the areas identified to have $10 \%$ slope or less were excluded.

\subsubsection{Service Area}

According to a survey that we conducted, most people in UP Diliman prefers to walk $200 \mathrm{~m}$ from their buildings for the sole purpose of buying food from the kiosk. To get areas $200 \mathrm{~m}$ from each building, Service Area Network Analysis was done. A road network was first created to be able to use the network analysis 
tools in ArcGIS. The buildings were set as Facilities and a buffer of 10 meters from the road was created. The tool was then used and the route buffer polygons produced were extracted. Road points that are not within these polygons were then excluded.

\subsubsection{Sheds and parking lots.}

Buildings, sheds and parking lots were considered in choosing the best candidates. Since the objective is to get equal customer turnout, the probability of customers must be maximized. Therefore, chosen candidates should be placed in areas where most people are present such as building sides and transportation areas like sheds and parking lots. Also, this is to be able to accommodate both sets of people that are commuting or driving cars.

A buffer of 5 meters from sheds and parking lots and 10 meters from buildings were used. Road points that are not within these buffers were also excluded. However, there are cases when some points were added or removed to maximize the probability of customers in the area.

\subsubsection{Closest Facility}

Now the reach of each candidate must then be identified. To do so, Closest Facility was used. Distances between the road points and the buildings were identified. However, all routes that exceed $200 \mathrm{~m}$ were removed to limit each road points to be associated with nearby buildings only. The remaining road points were used to identify which points in UP would reach the same demand.

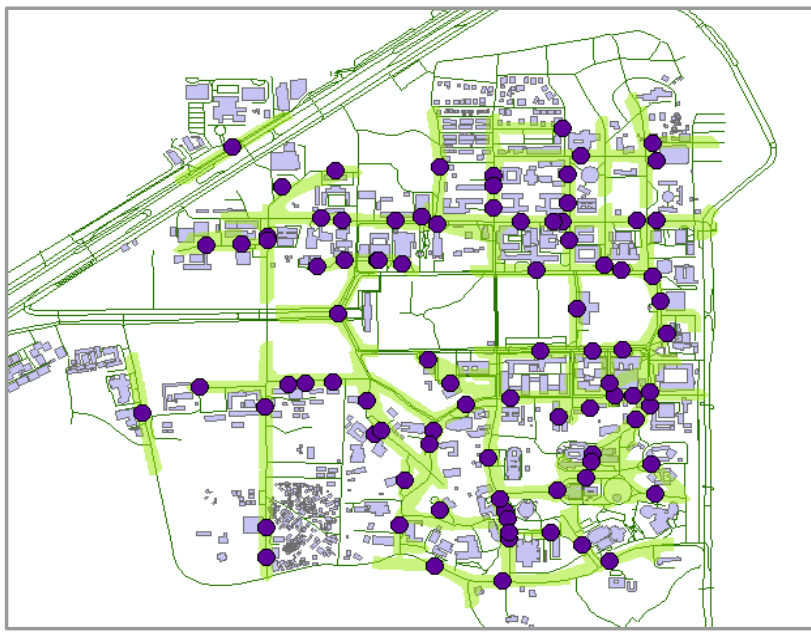

Figure 2. The product of the Service Area tool. Areas in green are the parts of the roads within 200 meters from each building.

\subsubsection{Python code}

As far as we know, ArcGIS does not provide a tool that iterates in order to identify equal demands. This leads us to create our own python code for iteration.

The code relates the weight to the inverse of the distance, which leads to a higher demand if the point is closer to the building.
Also, the demand will be distributed to points that provide for the same building. Demand distribution depends on the distance of the point to the building. The sum of these demands from all buildings reached by each point will be the weight of the point. The code then iterates until 20 points were found to have the least difference in weight

\section{RESULTS AND DISCUSSIONS}

The points identified by the code to have the least difference in probability were scattered within the UP Diliman Campus only. Thus, buildings like, AIT and those along CP Garcia has no nearby access to any kiosk. Also, the computed probability still has relatively high difference, considering that the probability of the lowest is half of that of the highest. This is due to the extreme uneven distribution of the population in UP Diliman and the short reach limited to only 200 meters.

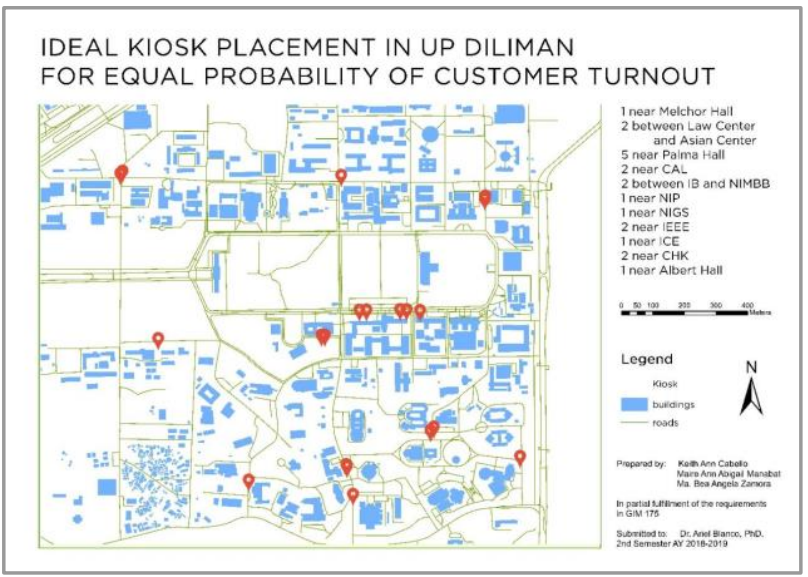

Figure 3. Map of the ideal kiosk locations.

As seen in Figure 3, five (5) kiosks were placed near Palma Hall. This is to accommodate the building with the highest population count in the campus. Two (2) more kiosk each were placed near CAL, between Law Center and Asian Center, near IEEE, and near CHK. One (1) kiosk each was placed near Melchor Hall, NIP, NIGS, ICE, and Albert Hall. With these placements, it is obviously evident that not all buildings will be reached by the kiosk, thus not having to serve $100 \%$ of the population. Tabulated in Table 1 is the locations of the kiosks and the buildings that each can reach within 200 meters.

Table 1. Kiosk Locations and their corresponding reach.

\begin{tabular}{|l|l|}
\hline Location & Reach \\
\hline 1 near NIP & NIP \\
\hline 2 near CAL & CAL, Vargas Museum \\
\hline 1 near Albert Hall & $\begin{array}{l}\text { Albert Hall, CFA, Office of the } \\
\text { Campus Architect, SPMO }\end{array}$ \\
\hline 2 near CHK & CSWCD, SOLAIR, SURP, CHK \\
\hline 1 near ICE & ICE, PAUW-UP \\
\hline
\end{tabular}




\begin{tabular}{|l|l|}
\hline 2 between IB and NIMBB & CS Admin, CSRC, IB, IC, NIMBB \\
\hline 2 near IEEE & $\begin{array}{l}\text { CS Lib, DMMME, IEEE, EnggLib } \\
\text { 2, NIGS, MSI }\end{array}$ \\
\hline 1 near NIGS & $\begin{array}{l}\text { CS Lib, DMMME, IEEE, EnggLib } \\
\text { 2, NIGS }\end{array}$ \\
\hline $\begin{array}{l}2 \text { between Law Center and } \\
\text { Asian Center }\end{array}$ & $\begin{array}{l}\text { Asian Center, Law Center, Malcolm } \\
\text { Hall, Econ }\end{array}$ \\
\hline 1 near Melchor & $\begin{array}{l}\text { Kalay, Melchor Hall complex, } \\
\text { Sanggumay, Computer Center }\end{array}$ \\
\hline 2 near Palma Hall & Palma Hall \\
\hline 3 near Palma Hall & Palma Hall, Benitez Hall, PH Annex \\
\hline
\end{tabular}

\section{CONCLUSION AND RECOMMENDATIONS}

Due to the uneven distribution of population in UP Diliman, it was difficult to identify locations that would have a relatively small difference in customer probability. The 20 points identified in the results were those with the least only which does not necessarily mean that the demand is equal.

Most of the locations of the points were along parking lots and sheds to accommodate people who are either commuting or driving cars. Some of the current locations of the kiosk are strategic enough to serve areas with high demand such as those near Palma Hall and CAL. Multiple kiosks are currently cramped in AS-CAL to provide for both buildings. Same with locations in CHK where currently there are two (2) kiosks placed there - one near Vanguard, and one near the jeepney stop. Also, there are kiosks already standing to provide for Melchor Hall, CFA, and ICE. However, there are locations identified that currently has no kiosk standing most especially in the CS complex. Demand is also high in this area since CS has one of the largest population in UP. Putting a kiosk in the area would give them high income.

\section{ACKNOWLEDGEMENTS}

Our group would like to thank our families for their unwavering support and understanding. We would also like to thank our friends and the UP Department of Geodetic Engineering for showering us with support throughout the entire duration of this research.

\section{REFERENCES}

Bojovic, et. al. (2005). Determining an Optimal Retail Location by Using GIS. Yugoslav Journal of Operations Research 16 (2006), Number 2, 253-264. Retrieved from http://elib.mi.sanu.ac.rs/files/journals/yjor/32/yujorn32p253264.pdf

Casinos, A., Saborit, G. (2015). Parametric Modeling of Human Gradient Walking for Predicting Minimum Energy Expenditure. Universitat de Barcelona: Departament de Biologia Animal.

\section{Retrieved} from https://www.hindawi.com/journals/cmmm/2015/407156/

Closest facility analysis.(n.d.). ArcGIS Pro: Network analysis layers.. Retrieved from https://pro.arcgis.com/en/proapp/help/analysis/networks/closest-facility-analysis-layer.htm

Dubuc, S. (2007). GIS-based accessibility analysis for network optimal location model: An application for bio-energy projects of the mineral industry in the UK. OpenEdition: European Journal of Geography. Retrieved from https://journals.openedition.org/cybergeo/12653?lang=en

Feng,M., et. al. (2007). Earthquake Disaster GIS and SpatialTemporal Pattern of Earthquake Disasters in China. Tsinghua University: School of Architecture. Retrieved from http://www.iitk.ac.in/nicee/wcee/article/14_07-0041.PDF

Garner, S. (2013). The top 7 factors for a good small business location. Go Forth Institute Online Small Business Training. Retrieved from http://canadianentrepreneurtraining.com/thetop-7-factors-for-a-good-small-business-location/

Gordon, K.M. (2017). Business Site Selection, Location Analysis, and GIS. Arcada University of Applied Sciences: International Business. Retrieved from https://www.theseus.fi/bitstream/handle/10024/140157/Thesis $\% 20$ Draft \%202.pdf?sequence $=1 \&$ is Allowed $=y$

Hodgson, M.E., Kar, B. (2008). A GIS-Based Model to Determine Site Suitability of Emergency Evacuation Shelters. University of South Carolina: Department of Geography. Transactions in GIS, 12(2): 227-248. Retrieved from https://onlinelibrary.wiley.com/doi/abs/10.1111/j.1467-

9671.2008.01097.x ?fbclid=IwAR2DvOIpnfVyB17F3X2tiOfSE nBE4cwyti7nWfEeAywXxftt_20tM5hUzjM\&

How Buffer (Analysis) works. (n.d.) ESRI: Proximity toolset. Retrieved

from http://desktop.arcgis.com/en/arcmap/10.3/tools/analysistoolbox/how-buffer-analysisworks.htm?fbclid=IwAR2KdtSa13Vm_KiPRwmRUN9986I1Jg 19UCA7LvCEOHGuQm67FbToiUVkjv4

Service area analysis. (n.d.). ESRI: Extensions. Retrieved from http://desktop.arcgis.com/en/arcmap/latest/extensions/networkanalyst/service-

area.htm\#ESRI_SECTION1_DEC185407C154056B6B17077F 9BAD8F2

Walkability Improvements: Strategies to Make Walking Convenient, Safe and Pleasant. (2017). TDM Encyclopedia: Victoria Transport Policy Institute. Retrieved from http://www.vtpi.org/tdm/tdm92.htm 